Western University

Scholarship@Western

Biology Publications

Biology Department

$1-1-2017$

\title{
Cold tolerance of third-instar Drosophila suzukii larvae.
}

Ruth Jakobs

Banafsheh Ahmadi

Sarah Houben

Tara D Gariepy

Brent J Sinclair

Follow this and additional works at: https://ir.lib.uwo.ca/biologypub

Part of the Biology Commons

Citation of this paper:

Jakobs, Ruth; Ahmadi, Banafsheh; Houben, Sarah; Gariepy, Tara D; and Sinclair, Brent J, "Cold tolerance of third-instar Drosophila suzukii larvae." (2017). Biology Publications. 85.

https://ir.lib.uwo.ca/biologypub/85 
1 Cold tolerance of third-instar Drosophila suzukii larvae

2

3 Ruth Jakobs $^{1 \dagger}$, Banafsheh Ahmadi ${ }^{2}$, Sarah Houben ${ }^{1,3}$, Tara D. Gariepy ${ }^{4}$ and Brent J. Sinclair ${ }^{1 \ddagger}$

4

$5 \quad{ }^{1}$ Department of Biology, University of Western Ontario, London, ON, Canada

$6 \quad{ }^{2}$ Department of Entomology, Faculty of Agriculture, Tarbiat Modares University, Tehran, Iran

$7 \quad{ }^{3}$ Institute of Zoophysiology, University of Münster (WWU), Münster, Germany

$8 \quad{ }^{4}$ Agriculture and Agri-Food Canada, Southern Crop Protection and Food Research Centre,

9 London, ON, Canada

10

$11{ }^{\dagger}$ Present address: Department of Chemical Ecology, Bielefeld University, Germany

12

$13 \stackrel{\ddagger}{\ddagger}$ Author for correspondence. Brent Sinclair, Department of Biology, University of Western

14 Ontario, London, ON, N6G 1L3, Canada. Email bsincla7@uwo.ca, tel. 519-661-2111 x83138,

15 fax 519-661-3935

16

17 
Drosophila suzukii is an emerging global pest of soft fruit; although it likely overwinters as an adult, larval cold tolerance is important both for determining performance during spring and autumn, and for the development of temperature-based control methods aimed at larvae. We examined the low temperature biology of third instar feeding and wandering larvae in and out of food. We induced phenotypic plasticity of thermal biology by rearing under short days and fluctuating temperatures $\left(5.5-19^{\circ} \mathrm{C}\right)$. Rearing under fluctuating temperatures led to much slower development (42.1 days egg-adult) compared to control conditions (constant $21.5^{\circ} \mathrm{C}$; $15.7 \mathrm{~d}$ ), and yielded larger adults of both sexes. D. suzukii larvae were chill-susceptible, being killed by low temperatures not associated with freezing, and freezing survival was not improved when ice formation was inoculated externally via food or silver iodide. Feeding larvae were more cold tolerant than wandering larvae, especially after rearing under fluctuating temperatures, and rearing under fluctuating temperatures improved survival of prolonged cold $\left(0^{\circ} \mathrm{C}\right)$ to beyond 72 $\mathrm{h}$ in both larval stages. There was no evidence that acute cold tolerance could be improved by rapid cold-hardening. We conclude that $D$. suzukii has the capacity to develop at low temperatures under fluctuating temperatures, but that they have limited cold tolerance. However, phenotypic plasticity of prolonged cold tolerance must be taken into account when developing low temperature treatments for sanitation of this species.

Keywords: spotted wing drosophila; cold tolerance; chill susceptible; overwintering; phenotypic plasticity; fluctuating thermal regimes 


\section{Introduction}

42 Spotted wing drosophila, Drosophila suzukii (Matsumura) (Diptera: Drosophilidae), is an emerging global pest of soft fruit (Cini et al., 2014; Lee et al., 2011; Walsh et al., 2010). D. suzukii lays eggs in unripe fruit. The entry wound and larval development promote fruit degradation, resulting in significant losses to blueberry, strawberry and cherry crops (Bolda et al., 2010). As with most Drosophila except $D$. lutescens, which may overwinter as a larva or pupa in Japan (Kimura, 1988), D. suzukii appears to overwinter as an adult, and there is a welldescribed 'winter morph' that is darker than the summer morph (Zerulla et al., 2015). This winter morph has some improved tolerances to environmental stress (Plantamp et al., 2016; Shearer et al., 2016; Toxopeus et al., 2016; Wallingford et al., 2016). However, larvae appear to be significantly less cold tolerant than adults, being killed by short exposures to sub-zero temperatures (Dalton et al., 2011) and longer exposures to temperatures near $0{ }^{\circ} \mathrm{C}$ (Kanzawa, 1939).

Insect cold tolerance strategies are usually divided into freeze tolerance (those that can withstand internal ice formation) and freeze avoidance, wherein individuals can survive cold as long as they do not freeze, but are killed when ice formation occurs (the supercooling point, SCP; Sinclair et al., 2015). The majority of insects, however, are chill-susceptible, killed by processes unrelated to ice formation at temperatures above the SCP (Sinclair et al., 2015). Strachan et al. (2011) found that larvae of 18 of 27 Drosophila were chill-susceptible, with another eight freezeavoidant. Larvae of the closely-related Chymomyza costata and C. amoena are freeze tolerant when sufficiently cold-acclimated and with external ice inoculation (Koštál et al., 2011; Sinclair et al., 2009). However, no Drosophila larvae are currently thought to be freeze tolerant. Cold tolerance can also be phenotypically plastic. D. melanogaster larvae exhibit a rapid cold- 
hardening response (Czajka and Lee, 1990), as well as responding to longer-term acclimation (Rajamohan and Sinclair, 2009).

We observed that some late-instar $D$. suzukii larvae in field cages survived a cold snap in November 2014 that reached $-6.9^{\circ} \mathrm{C}$ and killed all the adult flies. This led us to hypothesise that acclimation or hardening may make larvae more cold-tolerant than previously reported.

Moreover, because the host fruit are often exported, cold tolerance of the larvae is relevant for determining the capacity of larvae to survive chilling during processing and transport. Thus, our objective was to better characterise the cold tolerance of $D$. suzukii larvae. We measured growth and development, SCP, cold tolerance strategy and acute and chronic lethal temperatures of third-instar feeding and wandering larvae with and without an acclimation under fluctuating temperatures. For feeding larvae, we conducted experiments both within food (replicating likely field conditions) and without food (which allows us to better control the conditions and get a more precise measure of lethal limits). 9

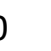

\section{Methods}

Animal rearing and treatment groups

We established a Drosophila suzukii population from approximately 200 individuals collected in the Halton Hills region, Ontario, Canada $\left(43^{\circ} 34^{\prime} \mathrm{N} 79^{\circ} 57^{\prime} \mathrm{W}\right)$. We reared flies on a bananacornmeal-agar medium (Markow and O'Grady, 2005), at $21.5 \pm 1^{\circ} \mathrm{C}$ and $60 \pm 5 \%$ relative humidity under 13:11 L:D, as described elsewhere (Jakobs et al., 2015; Nyamukondiwa et al., 2011; Toxopeus et al., 2016). We used 3.7 L population cages containing approximately 300 adult flies that were two to six days post-eclosion (to reduce any parental age effect). Flies laid eggs on Petri dishes of banana food that had been dyed green with food colouring, which allowed 
us to separate feeding and non-feeding larvae. We removed the plates from the population cages every 24 h, and reared larvae on the Petri dishes.

To induce phenotypic plasticity in $D$. suzukii larvae, we placed the food plates with the eggs into two different rearing conditions (treatment). Eggs were placed under either control conditions $\left(21.5^{\circ} \mathrm{C}, 13: 11 \mathrm{~L}: \mathrm{D}\right)$ or exposed to a fluctuating thermal regime (FTR; $5.5^{\circ} \mathrm{C} / 19{ }^{\circ} \mathrm{C}, 11.5: 12.5$ L:D), simulating the average photoperiod and daily minimum and maximum temperatures from late September in London, Ontario.

We used third instar feeding and wandering larvae for experiments. We checked the food plates for larvae on a daily basis and removed larvae with a soft paintbrush. Banana food medium was carefully removed from larvae with tap water and larvae were blotted dry with a tissue. The life stage of a subset of larvae on each collection day was identified using the morphology of the mouth hooks (Figure 1A-C) and anterior spiracles (Figure 1D), based upon Demerec’s (1965) descriptions for $D$. melanogaster. In addition, feeding third instar individuals appeared green as they still carried green food in their gut, while wandering-stage instars were transparent and lacked food in the gut (Figure 1E).

To determine the effect of the treatments on developmental time, eggs were reared into adults under control conditions, FTR or a constant low temperature (11 $\left.{ }^{\circ} \mathrm{C}, 10: 14 \mathrm{LD}\right)$. We removed pieces of the banana medium carrying approximately ten eggs, and transferred them into $35 \mathrm{~mL}$ vials containing banana medium ( $\mathrm{n}=6$ vials/treatment). We collected the adults that developed from these eggs daily and stored them at $-20{ }^{\circ} \mathrm{C}$. When emergence had ended, we 
112 dried the flies over silica gel for approximately $48 \mathrm{~h}$. Flies were sexed and weighed (MX5

113 microbalance, Mettler Toledo, Columbus, OH, USA) as a measure of offspring dry mass.

Cold tolerance

We determined cold tolerance parameters using the approach described by Sinclair et al. (2015).

117 To determine the supercooling point (SCP), we placed larvae individually into $1.7 \mathrm{~mL}$

118 microcentrifuge tubes in contact with a 36-AWG type-T copper-constantan thermocouple

119 (Omega, Laval, Quebec, Canada) connected to a computer via a TC-08 interface and Picolog v5.20.1 software (Pico Technology, Cambridge, UK), which recorded the temperature at $0.5 \mathrm{~s}$ intervals. The tubes were placed into holes in an aluminium block cooled by methanol (diluted c.

$12250 \%$ in water) circulated from a refrigerated bath (Lauda Proline 3530, Würzburg, Germany).

123 Larvae were equilibrated at $0{ }^{\circ} \mathrm{C}$ and cooled to $-30{ }^{\circ} \mathrm{C}$ at $0.1^{\circ} \mathrm{C} / \mathrm{min}$. The SCP was defined as 124 the lowest temperature before the exotherm caused by the latent heat of crystallisation.

To determine the cold tolerance strategy, larvae were placed into microcentrifuge tubes and cooled, as described for the SCPs. After half the larvae had frozen (indicated by the exotherm), 128 all individuals were removed quickly to room temperature and placed individually into the wells 129 of 6-well cell culture plates with a ca. $1 \mathrm{~cm}^{3}$ piece of banana food. Survival was assessed as the 130 ability to develop into adults. Flies were considered chill susceptible if both unfrozen and frozen 131 flies died, freeze-avoidant if all unfrozen flies survived (but those that froze died), and freeze 132 tolerant if individuals that froze survived.

134 Because some insects are freeze tolerant only with external ice inoculation, (e.g. Shimada and 135 Riihimaa, 1988) we applied an external ice nucleator (silver iodide) to initiate freezing (Strachan 
et al., 2011). We dipped larvae into a silver iodide/water slurry and determined the SCP and cold tolerance strategy as described above.

We estimated the acute lethal temperature (LT) of third feeding and wandering larvae of the control and FTR group by exposing these larvae to a range of low temperatures for $1 \mathrm{~h}$. Groups of ten larvae were placed into a $0.65 \mathrm{~mL}$ microcentrifuge tube ( $\mathrm{n}=3$ groups/ temperature/ stage/ treatment combination). These tubes were placed into a pre-cooled aluminium block (described above) and held for $1 \mathrm{~h}$ at temperatures ranging from $-15{ }^{\circ} \mathrm{C}$ to $0^{\circ} \mathrm{C}$ (encompassing $0-100 \%$ mortality). Temperature during exposure was recorded in two blank tubes that were directly placed next to the tubes with larvae in the cooling block using thermocouples as above. Following the low temperature exposure we placed each opened tube into a $35 \mathrm{~mL}$ vial containing banana medium and reared the larvae to eclosion under control conditions. Survival was determined as the ability to eclose as adult.

Because larvae might be exposed to low temperatures inside their food, we also determined acute low-temperature survival of larvae in $35 \mathrm{~mL}$ vials containing banana food. Groups of 20 larvae were placed into each vial, which was exposed to low temperatures for $1 \mathrm{~h}$ as described above. Temperature was determined by placing the thermocouples inside the food medium $(2 \mathrm{~cm}$ below the food surface). After cold exposure, food vials containing the larvae were placed under control conditions and the number of adults that eclosed counted as a measure of survival. The temperature measured inside and outside the food differs during a $1 \mathrm{~h}$ exposure. Thus, temperature was recorded inside and outside the food during an exposure to $-9{ }^{\circ} \mathrm{C}(\mathrm{n}=10)$. SCP of the banana food was determined during this exposure and used in analyses. 
To determine survival of prolonged exposure to milder cold temperatures (see Sømme (1996) for rationale), we placed groups of ten larvae into food vials ( $\mathrm{n}=3$ groups/ stage/ treatment/ time), and assessed survival after exposure for $6,12,18,24,36,48,60,72$ and $120 \mathrm{~h}$ to $0{ }^{\circ} \mathrm{C} / 60 \% \mathrm{RH}$ in a Tenney ETCU16 chamber (Thermal Product Solutions, White Deer, PA, USA). Survival was assessed as successful eclosion after the vial was returned to $21.5^{\circ} \mathrm{C}$.

To test for a rapid cold-hardening response, larvae were pre-exposed to $0{ }^{\circ} \mathrm{C}$ or $4{ }^{\circ} \mathrm{C}$ for one hour with one hour recovery at $21.5^{\circ} \mathrm{C}$ (cf. Ransberry et al., 2011) and survival was determined at temperatures close to the previously estimated $\mathrm{LT}_{80-1 h}$ (temperature at which $80 \%$ of the individuals die after $1 \mathrm{~h}$ exposure; control feeding $-4.6{ }^{\circ} \mathrm{C}$; FTR feeding: $-8.7^{\circ} \mathrm{C}$; control wandering: $-6.6^{\circ} \mathrm{C}$; FTR wandering $-8.8^{\circ} \mathrm{C}$ ). Ten larvae were placed into $0.65 \mathrm{~mL}$ microcentrifuge tubes, each tube was placed into a $50 \mathrm{~mL}$ vial, which was immersed in a cooling bath set to the $\mathrm{LT}_{80-1 \mathrm{~h}}$ for $1 \mathrm{~h}$ ( $\mathrm{n}=5$ groups/ stage/ treatment combination). After cold exposure, the tubes were placed into food vials kept under rearing conditions; survival was assessed as successful eclosion.

\section{Data analysis}

All analyses were conducted in in R version 3.0.1 (R Core Team, 2012). SCPs and dry mass were compared among treatments and stages or sex using a two-way ANOVA, for which model assumptions were checked. Survival after exposure to the $\mathrm{LT}_{80-1 \mathrm{~h}}$ was compared among treatments using Kruskal-Wallis test. We used accelerated failure time models (AFT) from the survival package in $\mathrm{R}$ to determine time at which $80 \%$ of the individuals developed from eggs into adults ( $\left.\mathrm{Dt}_{80}\right)$. The best-fit models used a log-logistic error distribution and treatment and stage as factors. Developmental time was compared among treatments using a Kuskal-Wallis test 
and between sex using a Wilcoxon rank sum test. The effect of the interaction of the treatments and sex was analysed with a Kruskal-Wallis test followed by a Wilcoxon pairwise comparison with Bonferroni-Holm correction.

The $\mathrm{LT}_{80-1 \mathrm{~h}}$ (temperature at which $80 \%$ of flies will die after a $1 \mathrm{~h}$ exposure) and Lt80 (lethal time at which $80 \%$ of the individuals die during chronic low-temperature exposure) were calculated for both third instar feeding and wandering larvae from the control and FTR groups via a generalized linear model (Venables and Ripley, 2002) with a binomial error distribution and logit link function (fit was tested with Wald's $\chi^{2}$ ) using the package MASS in R . Differences between groups were compared using a generalized linear model. We used the ghlt() function of the package multcomp in R (Bretz et al., 2011) to run a Tukey’s post-hoc comparison using the treatment $\times$ stage interaction.

\section{Results}

The rearing conditions altered the developmental time from egg to adult $\left(\right.$ Wald $\chi^{2}=1246.41, \mathrm{df}=$ 2, $\mathrm{p}<0.001$ ). The $\mathrm{DT}_{80}$ (time taken for $80 \%$ of the individuals to eclose as adults) was shortest in control flies (15.7 \pm 0.1 days), followed by FTR flies (42.1 \pm 0.5 days). Flies reared under constant low temperatures had the longest development time (62.4 \pm 0.6 days, Figure 2). Females were consistently heavier than males, and flies reared under FTR and constant low temperatures were larger than controls (Figure 3).

Supercooling points ranged from $-23.3^{\circ} \mathrm{C}$ in a wandering larva reared under FTR to $-7.3^{\circ} \mathrm{C}$ in a feeding control larva (Table 1). Feeding third-instar larvae had higher SCPs than wandering third-instar larvae $\left(\mathrm{F}_{1,176}=76.612, \mathrm{p}<0.001\right)$, and while FTR treatment led to a slight increase in SCP of feeding larvae, it did not change the SCP of the wandering stage (treatment $\times$ stage: 
$208 F_{1,176}=2.968, p=0.087$; Table 1). No larvae survived internal ice formation, indicating that they 209 are not freeze-tolerant. Further, larvae did not survive temperatures slightly above the SCP, 210 indicating that the flies are chill-susceptible (Table 1). Application of an external ice nucleator 211 (AgI) significantly increased the $\mathrm{SCP}\left(\mathrm{F}_{1,176}=127.098, \mathrm{p}<0.001\right)$, but did not lead to freeze 212 tolerance (Table 1). There was no significant interaction between external ice nucleation and 213 rearing conditions on SCP (treatment $\times$ AgI: $\mathrm{F}_{1,176}=0.114, \mathrm{p}=0.736$ ).

We determined acute low temperature tolerance of control and FTR feeding and wandering larvae by exposing them to a range of temperatures between -15 and $0{ }^{\circ} \mathrm{C}$ with food (in food vials) and without food (in tubes). The temperature inside the food decreased more slowly than outside the food, and the food froze at $-8.2 \pm 0.4{ }^{\circ} \mathrm{C}(\mathrm{n}=10$, example shown in Figure 3 froze at at $-8{ }^{\circ} \mathrm{C}$ after $\left.32 \mathrm{~min}\right)$. Overall survival decreased with the temperature. Acute low-temperature survival of larvae without food was affected by the treatment (Table 2). Feeding larvae of the FTR group survived lower temperatures than feeding larvae of the control group with no overlap of the survival curves (Figure 5A); whereas the survival curves of all the groups overlapped in wandering larvae (Figure 5B). In addition, survival was affected by the life stage (Table 2). The

$224 \mathrm{LT}_{80-1 \mathrm{~h}}$ (temperature at which $80 \%$ of the individuals die after $1 \mathrm{~h}$ exposure) was the lowest in 225 FTR feeding larvae $\left(-8.9 \pm 0.3^{\circ} \mathrm{C}\right)$ and FTR wandering larvae $\left(-8.4 \pm 0.4{ }^{\circ} \mathrm{C}\right)$, followed by the control wandering larvae $\left(-6.6 \pm 0.1{ }^{\circ} \mathrm{C}\right.$, Table 3). Control feeding larvae had the highest $\mathrm{LT}_{80-1 \mathrm{~h}}$ $227\left(-4.8 \pm 0.3{ }^{\circ} \mathrm{C}\right)$. Acute low-temperature survival determined with food was affected by the life 228 stage (Table 2). FTR feeding larvae and FTR wandering larvae had a lower LT $80-1 \mathrm{~h}$ (feeding: -9.6 $229 \pm 0.3^{\circ} \mathrm{C}$, wandering: $-8.7 \pm 0.3^{\circ} \mathrm{C}$ ) than control feeding and wandering larvae (feeding $-8.0 \pm 0.3$ $230{ }^{\circ} \mathrm{C}$, wandering: $-7.2 \pm 0.2{ }^{\circ} \mathrm{C}$ ) (Table 3). Survival curves of feeding larvae from different 231 treatments did not overlap (Figure 5C), whereas they did among groups of wandering larvae 
overlapped (Figure 5D). Feeding larvae show a lower $\mathrm{LT}_{80}$ when exposed to low temperatures with food than without food, whereas there is no difference for wandering larvae (Figure 5).

We checked larval survival after exposing them to $0^{\circ} \mathrm{C}$ for up to $120 \mathrm{~h}$. Mortality began after $6 \mathrm{~h}$ at $0{ }^{\circ} \mathrm{C}$ in both control and FTR wandering larvae and FTR feeding larvae, but after $12 \mathrm{~h}$ at 0 ${ }^{\circ} \mathrm{C}$ for control feeding larvae (Figure 6). However, mortality accumulated more slowly in FTR larvae: all the control wandering larvae died after $72 \mathrm{~h}$, whereas there was still some survival of FTR larvae at the $72 \mathrm{~h}$ timepoint (Figure 6; Table 2). Survival was affected by the interactions of time, treatment and life stage (except treatment $\times$ stage, Table 3).

To test for a rapid cold-hardening response, we exposed both FTR and control larvae to different pre-treatments followed by a $1 \mathrm{~h}$ exposure to a discriminating temperature. We did not observe any increase in acute cold tolerance by either larval stage under any rearing or pretreatment condition (Figure 7).

\section{Discussion}

Understanding low temperature survival by $D$. suzukii larvae could facilitate the development of temperature-based treatment of fruit or packaging for export, and reveals the potential for $D$. suzukii to overwinter in the larval stage, perhaps in waste fruit in orchards and vineyards. Here we show that third instar $D$. suzukii larvae are chill-susceptible, have limited plasticity of cold tolerance, and develop more slowly, but into larger adults, if reared under cool conditions.

Most insects follow a 'temperature-size rule' such that the rate of development increases, but body size decreases, with increasing temperature (Kingsolver and Huey, 2008). This appears to 
256 be true for $D$. melanogaster (Partridge et al., 1994), and our data show it is also the case for $D$. suzukii. Fluctuating temperatures are most consistent with the conditions experienced in nature, and development rate increases under FTR conditions, likely because of the effects of Jensen's inequality on development (Colinet et al., 2015). The outcomes of larval growth of Manduca sexta depend on both mean and fluctuations of temperature (Kingsolver et al., 2015), but our single fluctuating regime does not allow us to dissect these more subtle effects for $D$. suzukii. We did not determine whether this increased adult mass is due to increased energy reserves, as observed in adults from the $D$. auraria complex reared under fall conditions accumulated more triacylglycerol than summer morph flies (Ohtsu et al., 1993). If they do have increased energy stores, then this is likely due to acquisition during the larval period, since $D$. suzukii adults from this population that were transferred to fall-like conditions as wandering larvae did not have increased body size or triacylglycerol and carbohydrate content compared to those that developed under summer conditions (Toxopeus et al., 2016). Thus, the thermal sensitivity of larvae determines not only their cold tolerance, but also their potential performance as adults, and we speculate that in nature, the body size differences of the winter morphs likely results from larval responses, not the temperature/photoperiod effect.

Similar to adults of this species (Jakobs et al., 2015), both feeding and wandering $D$. suzukii larvae were chill susceptible, regardless of acclimation treatment or ice nucleation environment. Chill-susceptibility appears to be the ancestral state of cold tolerance for Drosophila, and is the only strategy reported in the melanogaster subgroup, to which D. suzukii belongs (Kimura, 2004;

277 Strachan et al., 2011). Chill susceptible insects are killed by both cold and freezing, so deliberate inoculation of ice formation is one possible way to enhance low temperature control of insects using this strategy (Strong-Gunderson et al., 1992). Because they are chill-susceptible, the SCP has limited ecological relevance (Sinclair et al., 2015), although changes in SCP can indicate modifications to gut contents (in this case perhaps explaining the shift in SCP with acclimation in 
282 feeding, but not wandering larvae), or to other physiological parameters (Coleman et al., 2014).

283 Larvae of some drosophilids survive internal ice formation only when it is inoculated externally 284 (e.g. by ice in the food; Shimada and Riihimaa, 1988), however we show that externally 285 inoculated freezing is lethal in D. suzukii, and freeze tolerance is therefore unlikely under natural 286 conditions, as well as in the lab.

Wandering larvae were more tolerant of acute cold exposure than feeding larvae, whereas the opposite was true during long-term exposure. Acute cold exposure likely causes direct injury to cells, while chronic cold exposure appears to be more related to long-term loss of homeostasis (MacMillan and Sinclair, 2011; Rajamohan and Sinclair, 2008; Sinclair and Roberts, 2005; Teets and Denlinger, 2013). The presence of food substantially increased acute low temperature survival in feeding larvae, possibly because the food may have substantially buffered the temperature exposure (Figure 4), effectively reducing the time for which feeding larvae were exposed to each temperature (Nedvěd et al., 1998). Wandering larvae have left the food, so even when food is present, they likely do not benefit from this buffering, which means that the presence of food cannot modify their tolerance. Feeding larvae tolerated $0{ }^{\circ} \mathrm{C}$ for approximately $40 \%$ longer than wandering larvae, which is surprising, since we would expect wandering larvae to be more resistant to environmental conditions - including temperature - since they have left the buffered environment of the food. Nevertheless, our results suggest that wandering larvae could be particularly susceptible to prolonged cold exposure, perhaps in the context of coldstorage of fruit.

Insects can increase their tolerance of low temperatures through plasticity via acclimation over long periods (including during development), or rapidly through hardening responses (Teets and Denlinger, 2013). Acclimation responses are usually especially robust under fluctuating temperature conditions (Colinet et al., 2015), including in adult D. suzukii (Jakobs et al., 2015). 
However, FTR acclimation had only a limited impact on acute cold tolerance, improving acute cold tolerance by less than $2{ }^{\circ} \mathrm{C}$ in feeding larvae when they were exposed to cold without food, but not modifying acute cold tolerance in other groups. Similarly, we did not detect a rapid coldhardening response in acute cold tolerance; however, we did not try a range of induction conditions, and it is possible that the $\mathrm{RCH}$ response is only elicited at lower temperatures (Sinclair and Chown, 2006). By contrast, FTR acclimation more than doubled survival time at 0 ${ }^{\circ} \mathrm{C}$ in both wandering and feeding larvae. Thus, although $D$. suzukii larvae appear to have limited plasticity for tolerance of absolute temperature, the limits for survival of long exposures are very plastic and need to be considered carefully when developing temperature-based treatments using mildly cold temperatures.

In conclusion, we show that $D$. suzukii larvae are not substantially cold tolerant, and that although there is plasticity in their tolerance to prolonged low temperatures, they have only limited ability to modify their acute cold tolerance. Thus, it could be possible to develop low temperature treatments that could control late-instar $D$. suzukii larvae without damaging fruit.

\section{Acknowledgements}

We are grateful to Iman Ashali, Daniel Ha, Andrew McLeod, Jantina Toxopeus and Halima Warsame for assistance in the lab, and to Justin Renkema, who supplied us with the D. suzukii population. This work was supported by the Natural Sciences and Engineering Research Council of Canada via a Discovery Grant to BJS, Agriculture and Agri-Food Canada Agriflex funding to TDG, and funding from Tarbiat Modares University to BA. Thanks to two anonymous reviewers, whose comments improved an earlier version of the manuscript. 
Bolda, M.P., Goodhue, R.E., Zalom, F.G., 2010. Spotted wing drosophila: potential economic impact of a newly established pest. Argric. Resource Econ. Update, 13, 5-8.

Bretz, F., Hothorn, T., Westfall, P.H., 2011. Multiple comparisons using R. CRC Press, New York.

Cini, A., Anfora, G., Escudero-Colomar, L.A., Grassi, A., Santosuosso, U., Seljak, G., Papini, A., 2014. Tracking the invasion of the alien fruit pest Drosophila suzukii in Europe. J. Pest Sci., 87, 559-566.

Coleman, P.C., Bale, J.S., Hayward, S.A.L., 2014. Cross-generation plasticity in cold hardiness is associated with diapause, but not the non-diapause developmental pathway, in the blow fly Calliphora vicina. J Exp Biol, 217, 1454-1461.

Colinet, H., Sinclair, B.J., Vernon, P., Renault, D., 2015. Insects in fluctuating thermal environments. Annu. Rev. Entomol., 60, 123-140.

Czajka, M.C., Lee, R.E., Jr., 1990. A rapid cold-hardening response protecting against cold shock injury in Drosophila melanogaster. J Exp Biol, 148, 245-254.

Dalton, D.T., Walton, V.M., Shearer, P.W., Walsh, D.B., Caprile, J., Isaacs, R., 2011. Laboratory survival of Drosophila suzukii under simulated winter conditions of the Pacific Northwest and seasonal field trapping in five primary regions of small and stone fruit production in the United States. Pest Manag. Sci., 67, 1368-1374.

Demerec, M., 1965. Biology of Drosophila. Hafner, New York. Jakobs, R., Gariepy, T.D., Sinclair, B.J., 2015. Adult plasticity of cold tolerance in a continentaltemperate population of Drosophila suzukii. J Insect Physiol, 79, 1-9.

Kanzawa, T., 1939. Studies on Drosophila suzukii Mats. Kofu. Yamanashi Agricultural Experimental Station, Yamanashi, Japan, pp. 1-49.

Kimura, M.T., 1988. Adaptations to temperate climates and evolution of overwintering strategies in the Drosophila melanogaster species group. Evolution, 42, 1288-1297.

Kimura, M.T., 2004. Cold and heat tolerance of drosophilid flies with reference to their latitudinal distributions. Oecologia, 140, 442-449.

Kingsolver, J.G., Higgins, J.K., Augustine, K.E., 2015. Fluctuating temperatures and ectotherm growth: distinguishing non-linear and time-dependent effects. J Exp Biol, 218, 2218-2225.

Kingsolver, J.G., Huey, R.B., 2008. Size, temperature, and fitness: three rules. Evol. Ecol. Res., 10, 251-268.

Koštál, V., Zahradnickova, H., Simek, P., 2011. Hyperprolinemic larvae of the drosophilid fly, Chymomyza costata, survive cryopreservation in liquid nitrogen. P Natl Acad Sci USA, 108, 13041-13046.

Lee, J.C., Bruck, D.J., Dreves, A.J., Ioriatti, C., Vogt, H., Baufeld, P., 2011. In Focus: Spotted wing drosophila, Drosophila suzukii, across perspectives. Pest Manag. Sci., 67, 1349-1351. MacMillan, H.A., Sinclair, B.J., 2011. The role of the gut in insect chilling injury: cold-induced disruption of osmoregulation in the fall field cricket, Gryllus pennsylvanicus. J Exp Biol, 214, 726-734.

Markow, T.A., O'Grady, P., 2005. Drosophila: A guide to species identification and use. Academic Press, London.

Nedvěd, O., Lavy, D., Verhoef, H.A., 1998. Modelling the time-temperature relationship in cold injury and effect of high-temperature interruptions on survival in a chill-sensitive collembolan. Funct. Ecol., 12, 816-824.

Nyamukondiwa, C., Terblanche, J.S., Marshall, K.E., Sinclair, B.J., 2011. Basal cold but not heat tolerance constrains plasticity among Drosophila species (Diptera: Drosophilidae). J. Evol. Biol., 24, 1927-1938. 
Ohtsu, T., Katagiri, C., Kimura, M.T., Hori, S.H., 1993. Cold Adaptations in Drosophila Qualitative Changes of Triacylglycerols with Relation to Overwintering. J. Biol. Chem., 268, 1830-1834.

Partridge, L., Barrie, B., Fowler, K., French, V., 1994. Evolution and development of body size and cell size in Drosophila melanogaster in response to temperature. Evolution, 48, 1269-1276. Plantamp, C., Salort, K., Gibert, P., Dumet, A., Mialdea, G., Mondy, N., Voituron, Y., 2016. All or nothing: Survival, reproduction and oxidative balance in Spotted Wing Drosophila (Drosophila suzukii) in response to cold. $J$ Insect Physiol, 89, 28-36.

$\mathrm{R}$ Core Team, 2012. R: A language and environment for statistical computing. R Foundation for Statistical Computing. www.R-project.org, Vienna.

Rajamohan, A., Sinclair, B.J., 2008. Short-term hardening effects on survival of acute and chronic cold exposure by Drosophila melanogaster larvae. J Insect Physiol, 54, 708-718. Rajamohan, A., Sinclair, B.J., 2009. Hardening trumps acclimation in improving cold tolerance of Drosophila melanogaster larvae. Physiol Entomol, 34, 217-223.

Ransberry, V.E., Macmillan, H.A., Sinclair, B.J., 2011. The relationship between chill-coma onset and recovery at the extremes of the thermal window of Drosophila melanogaster. Physiol. Biochem. Zool., 84, 553-559.

Shearer, P.W., West, J.D., Walton, V.M., Brown, P.H., Svetec, N., Chiu, J.C., 2016. Seasonal cues induce phenotypic plasticity of Drosophila suzukii to enhance winter survival. BMC Ecol., 16, 18.

Shimada, K., Riihimaa, A., 1988. Cold acclimation, inoculative freezing and slow cooling: essential factors contributing to the freezing-tolerance in diapausing larvae of Chymomyza costata (Diptera: Drosophilidae). Cryo-Lett, 9, 5-10.

Sinclair, B.J., Chown, S.L., 2006. Rapid cold-hardening in a Karoo beetle, Afrinus sp. Physiol Entomol, 31, 98-101.

Sinclair, B.J., Coello Alvarado, L.E., Ferguson, L.V., 2015. An invitation to measure insect cold tolerance: Methods, approaches, and workflow. J Therm Biol, 53, 180-197.

Sinclair, B.J., Gibbs, A.G., Lee, W.K., Rajamohan, A., Roberts, S.P., Socha, J.J., 2009.

Synchrotron X-Ray Visualisation of Ice Formation in Insects during Lethal and Non-Lethal Freezing. Plos One, 4, E8259.

Sinclair, B.J., Roberts, S.P., 2005. Acclimation, shock and hardening in the cold. J Therm Biol, 30, 557-562.

Sømme, L., 1996. The effect of prolonged exposures at low temperatures in insects. Cryo-Lett, 17, 341-346.

Strachan, L.A., Tarnowski-Garner, H.E., Marshall, K.E., Sinclair, B.J., 2011. The Evolution of Cold Tolerance in Drosophila Larvae. Physiol. Biochem. Zool., 84, 43-53.

Strong-Gunderson, J.M., Lee, R.E., Jr., Lee, M.R., 1992. Topical application of ice-nucleatingactive bacteria decreases insect cold tolerance. Appl. Env. Microbiol., 58, 2711-2716.

Teets, N.M., Denlinger, D.L., 2013. Physiological mechanisms of seasonal and rapid coldhardening in insects. Physiol Entomol, 38, 105-116.

Toxopeus, J., Jakobs, R., Ferguson, L.V., Gariepy, T.D., Sinclair, B.J., 2016. Reproductive arrest and stress resistance in winter-acclimated Drosophila suzukii. J Insect Physiol, 89, 37-51. Venables, W.N., Ripley, B.D., 2002. Modern Applied Statistics with S, 4th Edition ed. Springer, New York.

Wallingford, A.K., Lee, J.C., Loeb, G.M., 2016. The influence of temperature and photoperiod on the reproductive diapause and cold tolerance of spotted-wing drosophila, Drosophila suzukii. Entomol. Exp. Appl., 159, 327-337.

Walsh, D.B., Bolda, M.P., Goodhue, R.E., Dreves, A.J., Lee, J.C., 2010. Drosophila suzukii (Diptera: Drosophilidae): invasive pest of ripening soft fruit expanding its geographic range and damage potential. J. Integr. Pest Manag., 2, G1-G7. 
431 Zerulla, F.N., Schmidt, S., Streitberger, M., Zebitz, C.P.W., Zelger, R., 2015. On the 432 overwintering ability of Drosophila suzukii in South Tyrol. J. Berry Res., 5, 41-48.

433

434 
Figure 1. Identification of larval stages of Drosophila suzukii. Mouthparts of first (A), second (B), and third (C) larval instars vary in size and shape (scale bar: $50 \mu \mathrm{m}$ ). Third-instar wandering larvae (D) have well-developed anterior spiracles (scale bar $500 \mu \mathrm{m}$ ), while third-instar feeding larvae do not (not shown). Dyed food (green in colour, appears dark in figure) is apparent in the gut of larvae that are feeding, whereas third-instar wandering larvae have cleared their gut and 441 are transluscent (E).

Figure 2. Distribution of developmental time of Drosophila suzukii during different

treatments. Histograms of developmental time of females (A) and males (B) reared under control conditions (white; $21.5^{\circ} \mathrm{C}, 13: 11 \mathrm{~L}: \mathrm{D}$ ), fluctuating thermal regime (= FTR; light grey; $\left.5.5^{\circ} \mathrm{C} / 19^{\circ} \mathrm{C}, 11.5: 12.5 \mathrm{~L}: \mathrm{D}\right)$ and constant low temperatures (dark grey; $\left.11^{\circ} \mathrm{C}, 10: 14 \mathrm{~L}: \mathrm{D}\right)$. The $\mathrm{DT}_{80}$ (time at which $80 \%$ of the flies eclosed, see text for details) for the control group is represented by the dotted line, for the FTR group by the dashed line and for the constant low temperatures by the solid line. Lines with different letters denote significantly different developmental times across both A and B (treatment: $\chi^{2}=265.48, \mathrm{p}<0.001$, sex: $\mathrm{W}=14510, \mathrm{p}=$ 0.07 , treatment $\times$ sex: $\left.\chi^{2}=268.37, \mathrm{p}<0.001\right)$.

Figure 3. Weight of adult Drosophila suzukii reared under different treatments. Dry mass \pm SE (mg) was affected by sex and treatment of the flies (Treatment $F_{2,314}=4.437, p<0.05$, Sex: $F_{1,314}=119.551, p<0.001$, Treatment $\times$ Sex: $\left.F_{1,314}=0.65, p=0.523\right)$. Tukey’s HSD was run without the interaction, because it was non-significant. 
Figure 3. Differences in temperatures exposure inside and outside the food. The temperature exposure during 1 hour at $-9{ }^{\circ} \mathrm{C}$ in a food vial inside the food (dashed line) and outside the food (solid line).

Figure 5. Survival during acute low-temperature exposure of $D$. suzukii larvae. Larvae reared under control $\left(21.5^{\circ} \mathrm{C}, 13: 11 \mathrm{~L}: \mathrm{D}\right)$ conditions or a fluctuating thermal regime (=FTR; 5.5 $\left.{ }^{\circ} \mathrm{C} / 19{ }^{\circ} \mathrm{C}, 11.5: 12.5 \mathrm{~L}: \mathrm{D}\right)$ were exposed to a range of temperatures without food (A: feeding; B: wandering) or with food (C: feeding; D: wandering). The size of the symbols reflects the number of measurements of each group at this temperature (tubes: group of 10 larvae, food vial: group of 20 larvae, control = open symbols, FTR = crossed symbols). The dashed (control) and the solid (FTR) lines are the survival curve calculated with a generalized linear model (see Table 3 for statistics). The dotted line shows $80 \%$ mortality ( $\left.\mathrm{LT}_{80-1 \mathrm{~h}}\right)$. The grey box in $\mathrm{C}$ and $\mathrm{D}$ represent the mean SCP of the food \pm SE.

\section{Figure 6. Survival during chronic cold exposure of $D$. suzukii third instar larvae. Larvae} reared under control conditions $\left(21.5^{\circ} \mathrm{C}, 13: 11 \mathrm{~L}: \mathrm{D}\right)$ or a fluctuating thermal regime (FTR; 5.5 $\left.{ }^{\circ} \mathrm{C} / 19^{\circ} \mathrm{C}, 11.5: 12.5 \mathrm{~L}: \mathrm{D}\right)$ were exposed to $0{ }^{\circ} \mathrm{C}$ for up to $120 \mathrm{~h}$ (A: feeding; B: wandering). The size of the symbols reflects the number of measurements of each group at this time point ( $\mathrm{n}=3$ groups of 10, control = open symbols, FTR = crossed symbols). The dashed (control) and the solid (FTR) lines are the survival curve calculated with a generalized linear model (see Table 4 for statistics). The dotted line shows $80 \%$ mortality (Lt80).

\section{Figure 7. Survival following different short-term-hardening pre-treatments of $D$. suzukii} larvae. Third feeding and wandering larvae that were reared under control conditions $\left(21.5^{\circ} \mathrm{C}\right.$, 13:11 L:D; A: feeding, C: wandering) or a fluctuating thermal regime (= FTR; $5.5^{\circ} \mathrm{C} / 19{ }^{\circ} \mathrm{C}$, 
484 11.5:12.5 L:D; B: feeding, D: wandering) were pre-exposed to $0^{\circ} \mathrm{C}$ or $4{ }^{\circ} \mathrm{C}$ with one hour

485 recovery at $21.5^{\circ} \mathrm{C}$ and then exposed to temperatures close to the $\mathrm{LT}_{80-1 \mathrm{~h}}$ (control feeding to -4.6

$486{ }^{\circ} \mathrm{C}$, FTR feeding to $-8.7^{\circ} \mathrm{C}$, control wandering to $-6.6^{\circ} \mathrm{C}$ and FTR wandering to $-8.8^{\circ} \mathrm{C}$ ). There

487 was no difference in survival among any of the treatment groups. 
Table 1. Supercooling points and cold tolerance strategy of third instar larvae of Drosophila suzukii. Mean \pm SEM (sample size in parentheses). Control larvae were reared under $21.5^{\circ} \mathrm{C}, 13: 11 \mathrm{~L}: \mathrm{D}$, FTR (fluctuating thermal regime) under $5.5{ }^{\circ} \mathrm{C} / 19{ }^{\circ} \mathrm{C}, 11.5: 12.5 \mathrm{~L}: \mathrm{D} . \quad$ Silver test); see text for statistics. See text for rationale for determining cold tolerance strategies.

\begin{tabular}{|c|c|c|c|c|c|c|c|c|}
\hline \multirow{3}{*}{ Group } & \multicolumn{4}{|c|}{ Feeding } & \multicolumn{4}{|c|}{ Wandering } \\
\hline & \multirow{2}{*}{$\operatorname{SCP}\left({ }^{\circ} \mathbf{C}\right)$} & \multicolumn{2}{|c|}{ Number of flies dead } & \multirow{2}{*}{$\begin{array}{c}\text { Cold tolerance } \\
\text { strategy }\end{array}$} & \multirow{2}{*}{$\operatorname{SCP}\left({ }^{\circ} \mathrm{C}\right)$} & \multicolumn{2}{|c|}{ Number of flies dead } & \multirow{2}{*}{$\begin{array}{c}\text { Cold tolerance } \\
\text { strategy }\end{array}$} \\
\hline & & unfrozen & frozen & & & unfrozen & frozen & \\
\hline Control & $\begin{array}{c}-17.6 \pm 0.6^{b, c} \\
(n=35)\end{array}$ & $5 / 5$ & $5 / 5$ & chill-susceptible & $\begin{array}{c}-19.6 \pm 0.4^{\mathrm{c}} \\
(\mathrm{n}=27)\end{array}$ & $5 / 5$ & $5 / 5$ & chill-susceptible \\
\hline FTR & $\begin{array}{c}-15.1 \pm 0.7^{b} \\
(n=23)\end{array}$ & $5 / 5$ & $5 / 5$ & chill-susceptible & $\begin{array}{c}-20.6 \pm 0.5^{c} \\
(\mathrm{n}=22)\end{array}$ & $5 / 5$ & $5 / 5$ & chill-susceptible \\
\hline $\mathrm{FTR}+\mathrm{AgI}$ & $\begin{array}{c}-8.5 \pm 0.7^{\mathrm{a}} \\
(\mathrm{n}=18)\end{array}$ & $5 / 5$ & $5 / 5$ & chill-susceptible & $\begin{array}{c}-14.8 \pm 1.4^{\mathrm{b}} \\
(\mathrm{n}=17)\end{array}$ & $5 / 5$ & $5 / 5$ & chill-susceptible \\
\hline
\end{tabular}

493 
Table 2. Mortality after acute and prolonged low-temperature exposure for third feeding and wandering larvae of $\mathrm{D}$. suzukii. $\mathrm{LT}_{80}\left({ }^{\circ} \mathrm{C}\right.$,

\begin{tabular}{|c|c|c|c|c|c|c|c|c|}
\hline & \multirow{3}{*}{ Group } & \multirow{3}{*}{ Treatment } & \multicolumn{3}{|c|}{ Feeding larvae } & \multicolumn{3}{|c|}{ Wandering larvae } \\
\hline & & & \multirow{2}{*}{$\begin{array}{c}\mathbf{L T}_{80} / \\
\text { Lt80 }\end{array}$} & \multicolumn{2}{|c|}{ curve fit } & \multirow{2}{*}{$\begin{array}{c}\mathbf{L T}_{80} / \\
\mathbf{L t}{ }_{80}\end{array}$} & \multicolumn{2}{|c|}{ curve fit } \\
\hline & & & & Wald $\chi^{2}$ & $\mathbf{P}$ & & $\begin{array}{c}\text { Wald } \\
\chi^{2}\end{array}$ & $\mathbf{p}$ \\
\hline \multirow{4}{*}{$\mathbf{L T}_{80-1 h}\left({ }^{\circ} \mathrm{C}\right)$} & \multirow{2}{*}{ without food } & Control & $-4.8 \pm 0.3^{\mathrm{a}}$ & 6.63 & $<0.001$ & $-6.6 \pm 0.1^{b}$ & 5.52 & $<0.001$ \\
\hline & & FTR & $-8.9 \pm 0.3^{c}$ & 6.65 & $<0.001$ & $-8.4 \pm 0.4^{\mathrm{c}}$ & 5.75 & $<0.001$ \\
\hline & \multirow{2}{*}{ with food } & Control & $-8.0 \pm 0.3^{\mathrm{A}}$ & 10.29 & $<0.001$ & $-7.2 \pm 0.2^{\mathrm{B}}$ & 8.67 & $<0.001$ \\
\hline & & FTR & $-9.6 \pm 0.3^{\mathrm{C}}$ & 9.86 & $<0.001$ & $-8.7 \pm 0.3^{C}$ & 11.73 & $<0.001$ \\
\hline \multirow{2}{*}{ Lt80 (h) at $0^{\circ} \mathrm{C}$} & \multirow{2}{*}{ with food } & Control & $43.4 \pm 2.9^{\mathrm{a}}$ & 8.62 & $<0.001$ & $30.7 \pm 1.94^{\mathrm{a}}$ & -7.72 & $<0.001$ \\
\hline & & FTR & $92.2 \pm 7.2^{b}$ & 7.83 & $<0.001$ & $73 \pm 5.2^{b}$ & -8.28 & $<0.001$ \\
\hline
\end{tabular}



of third feeding and wandering larvae of $D$. suzukii reared under different conditions.

The generalized linear model was calculated with a binomial error distribution and logit link

503 function (fit was tested with Wald's $\chi^{2}$ ). Bold $P$-values indicate a significant effect of the

504 model term on survival. Treatments are rearing under control conditions $\left(21.5^{\circ} \mathrm{C}, 13: 11 \mathrm{~L}: \mathrm{D}\right)$

505 or a fluctuating thermal regime (FTR; $\left.5.5^{\circ} \mathrm{C} / 19{ }^{\circ} \mathrm{C}, 11.5: 12.5 \mathrm{~L}: \mathrm{D}\right)$, and we used two life

506 stages, feeding and wandering $3^{\text {rd }}$ instar larvae.

\begin{tabular}{|c|c|c|c|c|}
\hline \multirow{3}{*}{$\begin{array}{l}\text { Term } \\
\text { Acute cold model }\end{array}$} & \multicolumn{4}{|c|}{ Group } \\
\hline & \multicolumn{2}{|c|}{ without food } & \multicolumn{2}{|c|}{ with food } \\
\hline & Wald $\chi^{2}$ & $\boldsymbol{P}$ & Wald $\chi^{2}$ & $\boldsymbol{P}$ \\
\hline Temperature & 6.63 & $<0.001$ & 11.586 & $<0.001$ \\
\hline Treatment & 2.77 & $<0.01$ & 5.075 & 0.222 \\
\hline Life stage & 4.23 & $<0.001$ & 6.231 & $<0.01$ \\
\hline Temperature $\times$ Treatment & 0.47 & 0.636 & 3.003 & 0.578 \\
\hline Temperature $\times$ Life stage & 3.26 & $<0.01$ & 5.852 & $<0.01$ \\
\hline Treatment $\times$ Life stage & 3.75 & $<0.001$ & 5.962 & $<0.01$ \\
\hline Temperature $\times$ Treatment $\times$ Life stage & 2.9 & $<0.01$ & 5.344 & $<0.01$ \\
\hline \multicolumn{5}{|l|}{ Chronic cold model } \\
\hline Time & & & 8.62 & $<0.001$ \\
\hline Treatment & & & 0.37 & 0.713 \\
\hline Life stage & & & 1.99 & $<\mathbf{0 . 0 5}$ \\
\hline Time $\times$ Treatment & & & 3.88 & $<0.001$ \\
\hline Time $\times$ Life stage & & & 3.12 & $<\mathbf{0 . 0 1}$ \\
\hline Treatment $\times$ Life stage & & & 1.73 & 0.084 \\
\hline Time $\times$ Treatment $\times$ Life stage & & & 2.52 & $<0.05$ \\
\hline
\end{tabular}

507 
$510 \quad$ Figure 1

A: First larval instar
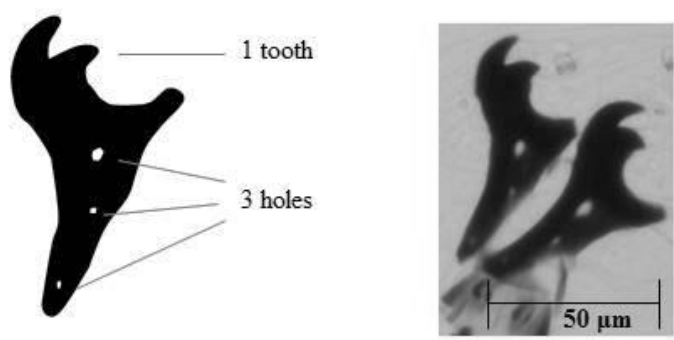

B: Second larval instar
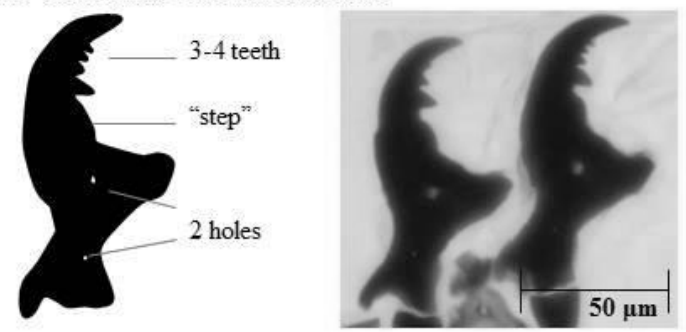

C: Third larval instar
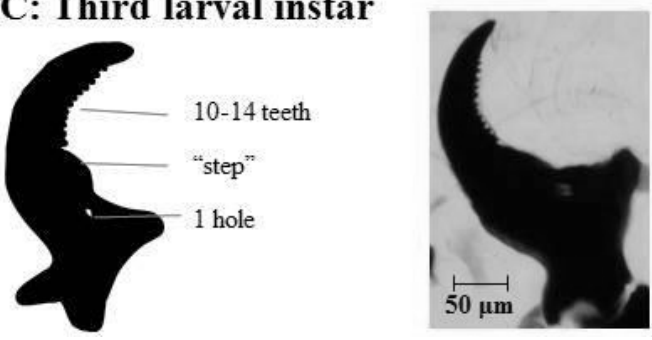

\section{D: Third larval instar (wandering)}

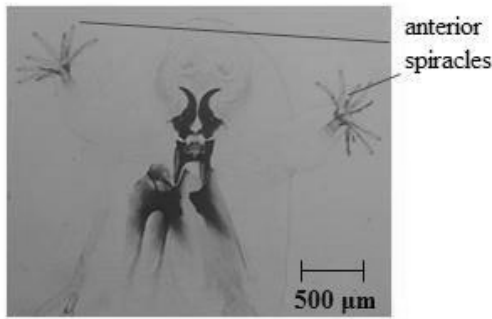

E: Wandering and feeding third larval instars

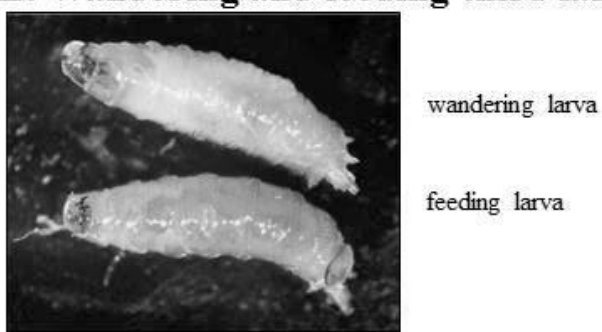

511 


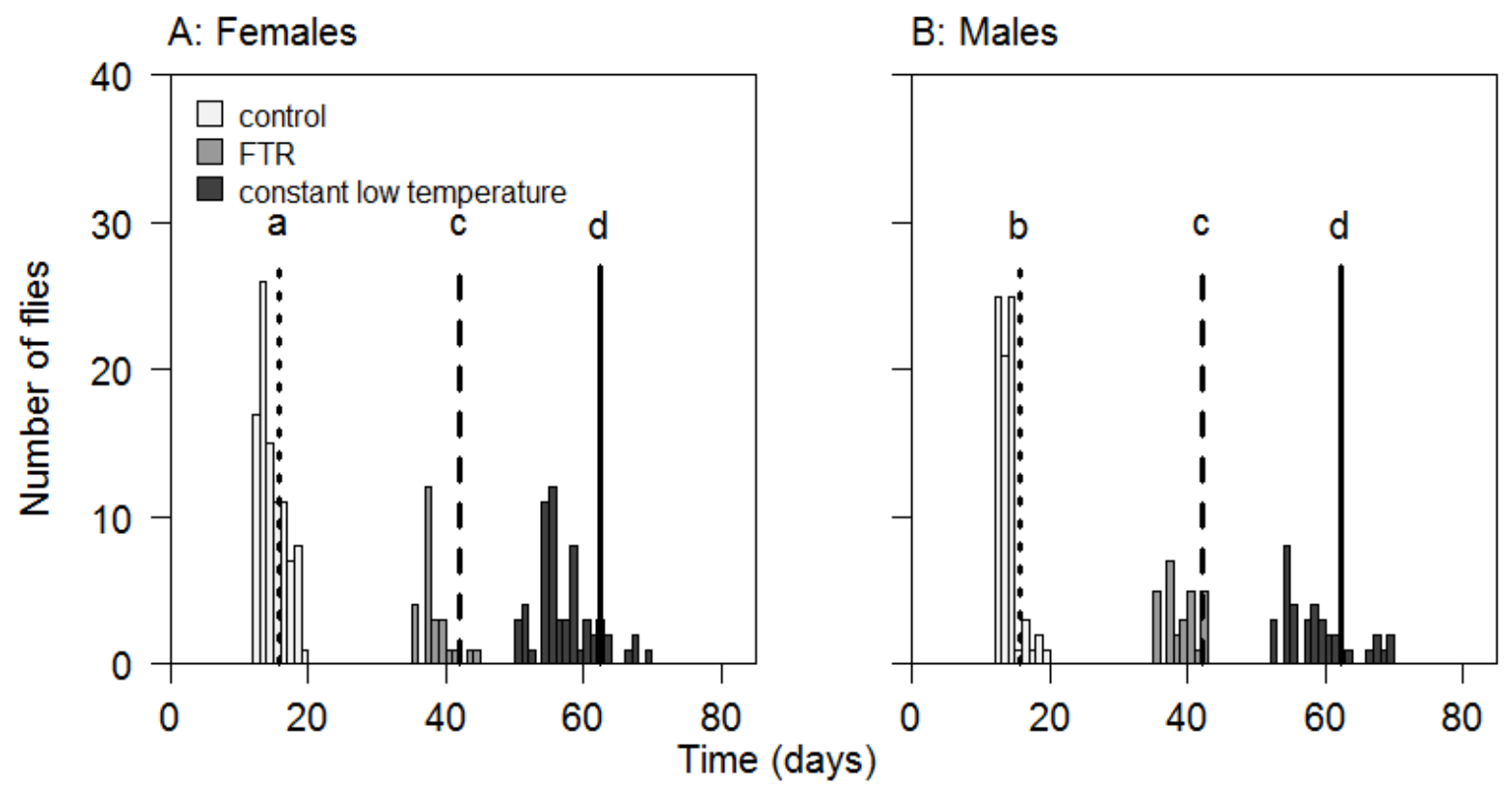


$515 \quad$ Figure 3

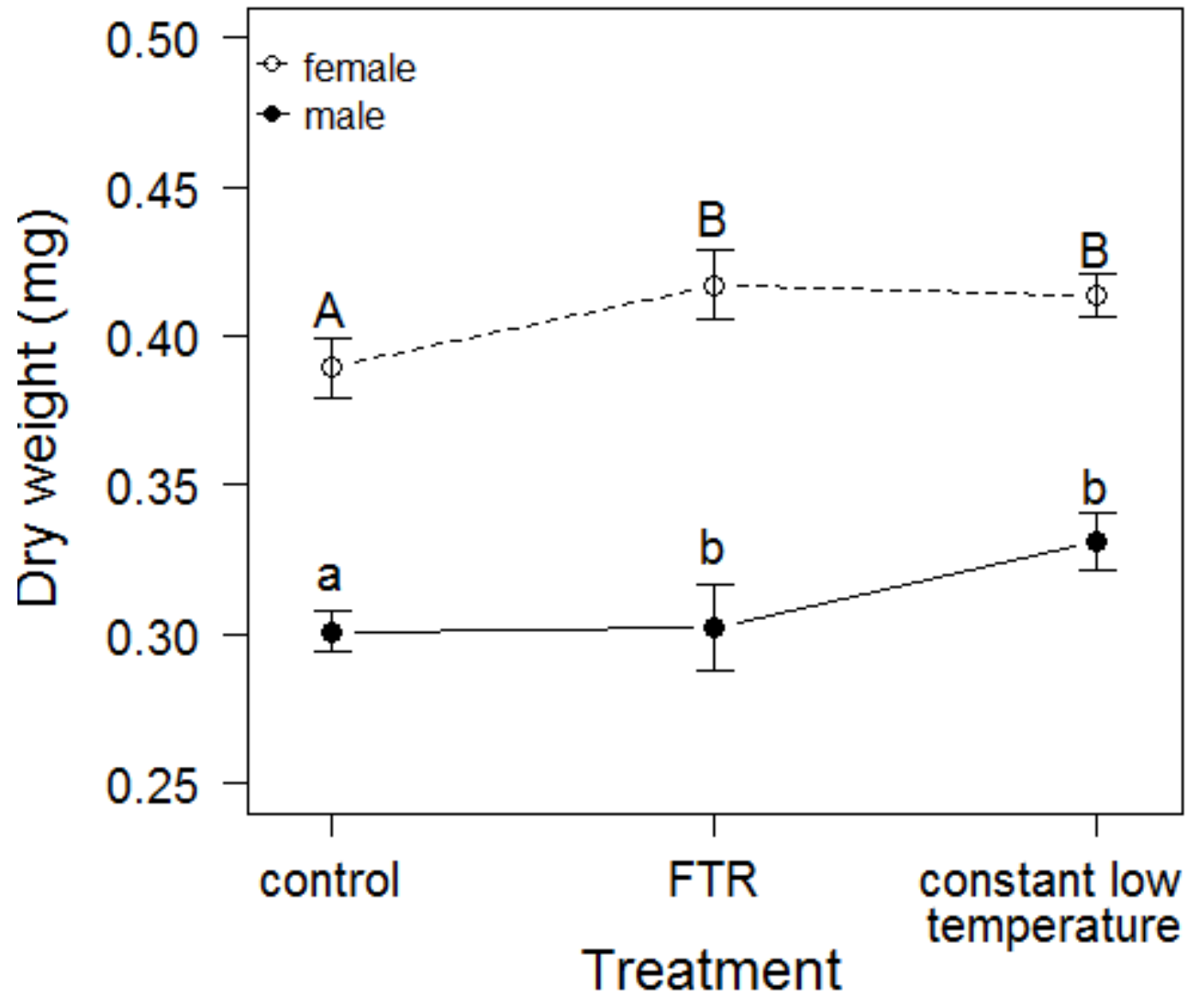

517 
Figure 4

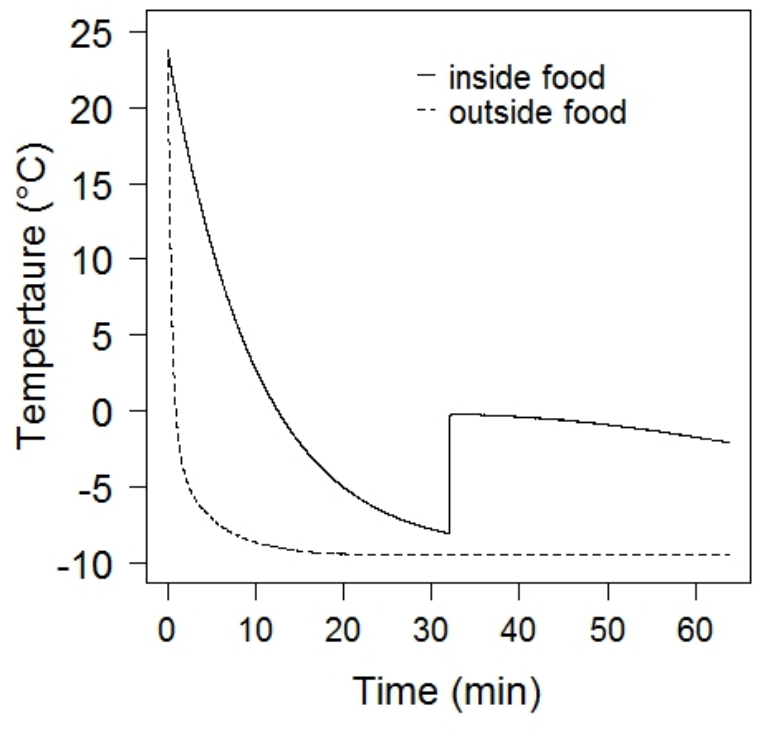

519

520

521 
A: Feeding larvae without food

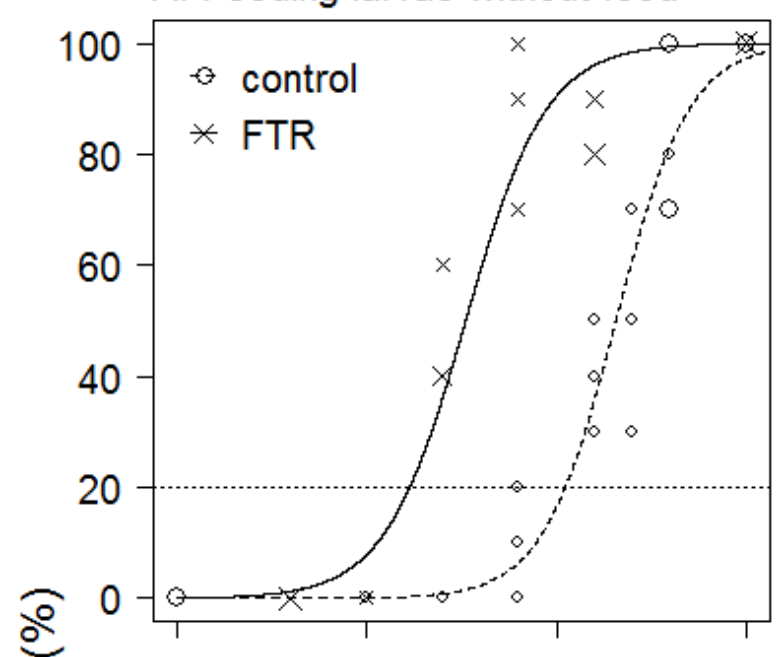

C: Feeding larvae with food

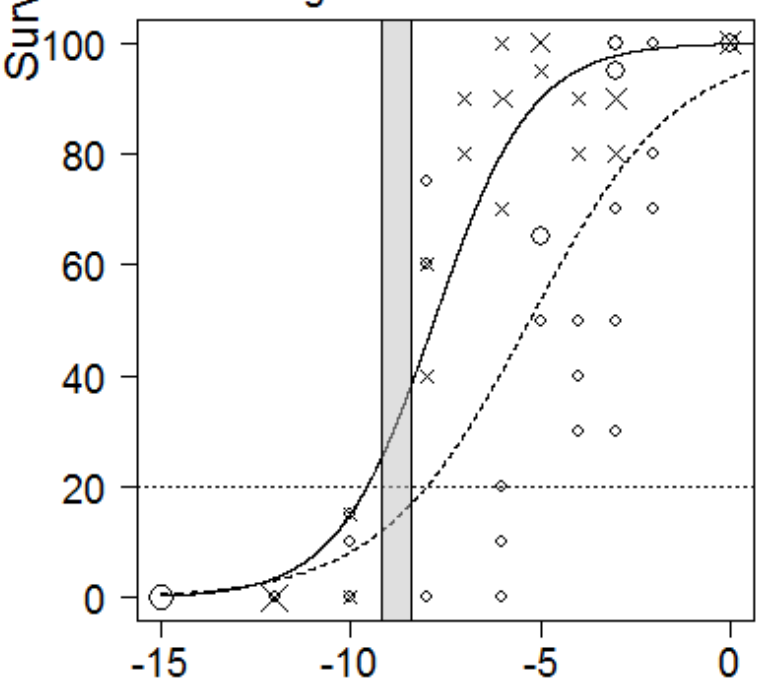

B: Wandering larvae without food

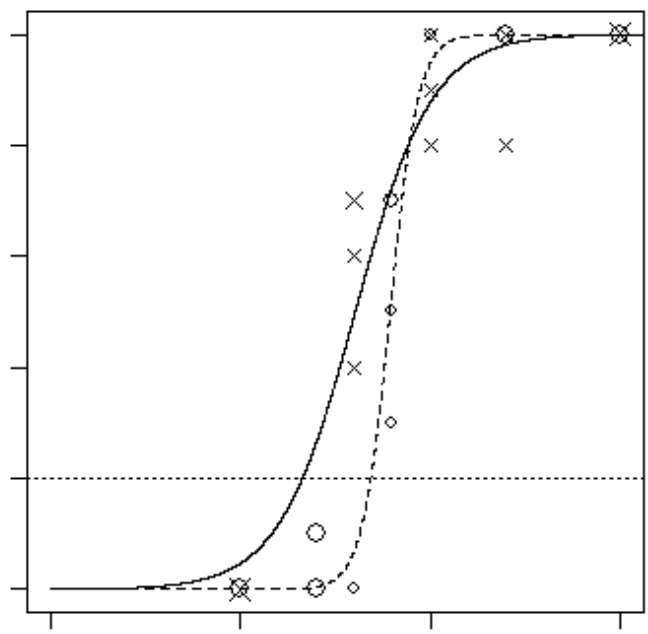

D: Wandering larvae with food

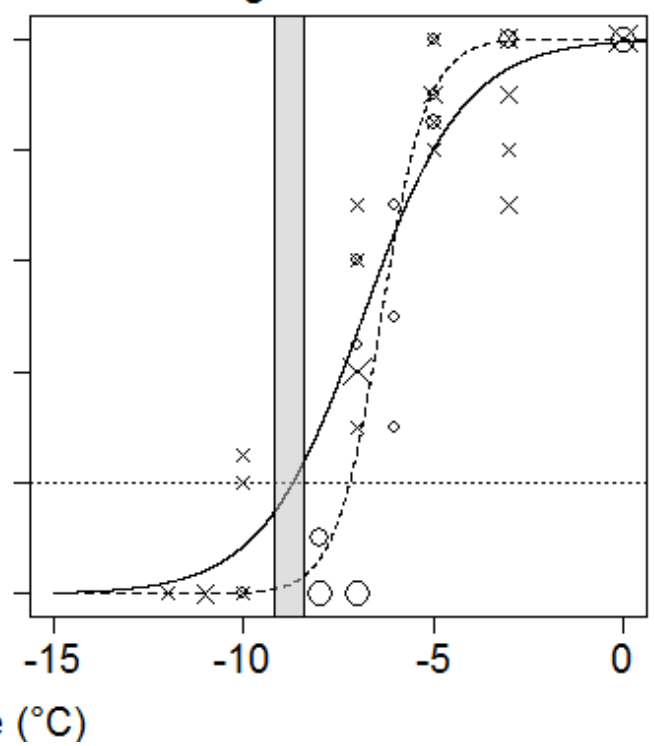




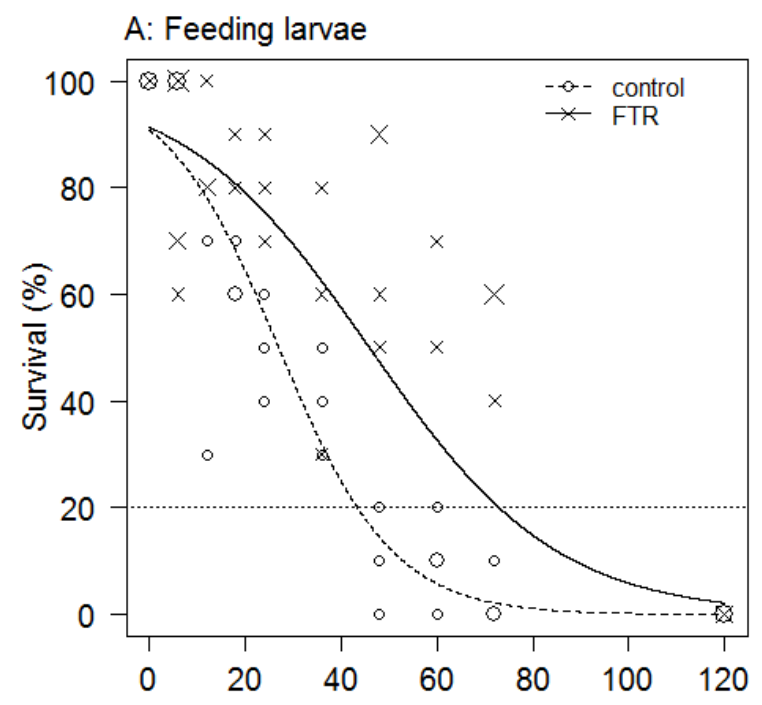

B: Wandering larvae

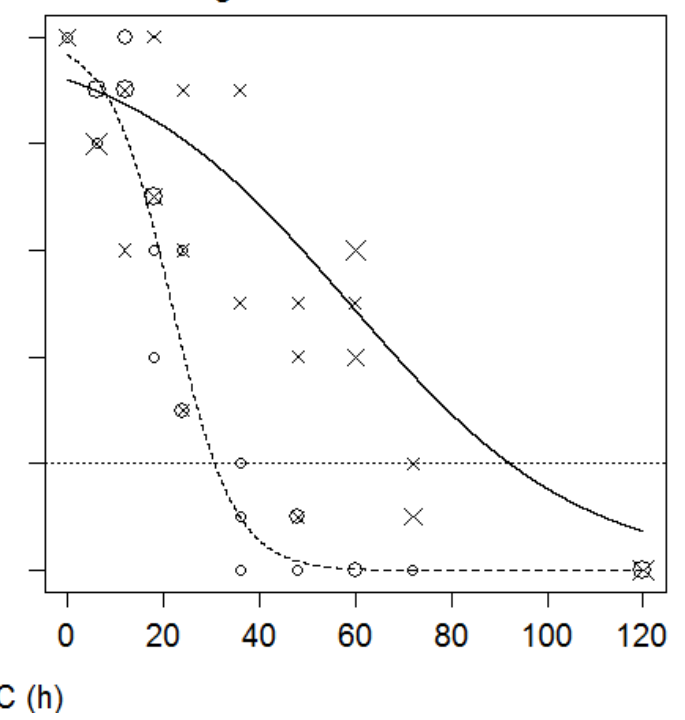

527 


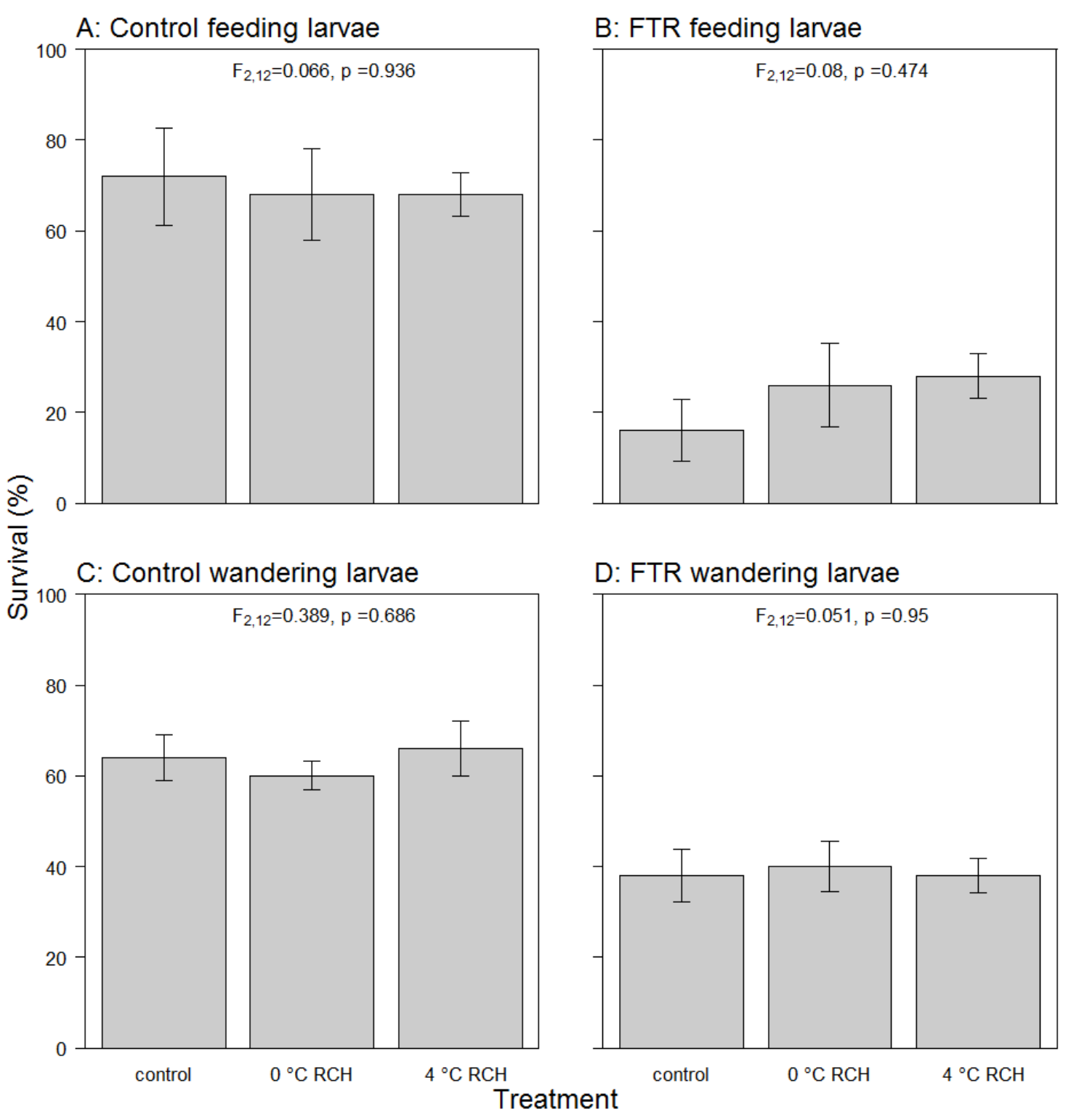

530 